\title{
Perspectives and Experiences of Transgender and Non-binary Individuals on Seeking Urological Care.
}

\author{
Paul H Chung \\ Thomas Jefferson University \\ Sabina Spigner \\ Thomas Jefferson University \\ Vishal Swaminathan \\ Thomas Jefferson University \\ Seth Teplitsky \\ Thomas Jefferson University \\ Rosemary Frasso \\ Thomas Jefferson University \\ Follow this and additional works at: https://jdc.jefferson.edu/urologyfp \\ Part of the Urology Commons \\ Let us know how access to this document benefits you
}

\section{Recommended Citation}

Chung, Paul H; Spigner, Sabina; Swaminathan, Vishal; Teplitsky, Seth; and Frasso, Rosemary, "Perspectives and Experiences of Transgender and Non-binary Individuals on Seeking Urological Care." (2020). Department of Urology Faculty Papers. Paper 63.

https://jdc.jefferson.edu/urologyfp/63

This Article is brought to you for free and open access by the Jefferson Digital Commons. The Jefferson Digital Commons is a service of Thomas Jefferson University's Center for Teaching and Learning (CTL). The Commons is a showcase for Jefferson books and journals, peer-reviewed scholarly publications, unique historical collections from the University archives, and teaching tools. The Jefferson Digital Commons allows researchers and interested readers anywhere in the world to learn about and keep up to date with Jefferson scholarship. This article has been accepted for inclusion in Department of Urology Faculty Papers by an authorized administrator of the Jefferson Digital Commons. For more information, please contact: JeffersonDigitalCommons@jefferson.edu. 
Perspectives and Experiences of Transgender and Non-binary Individuals on Seeking Urological Care

Paul H. Chung ${ }^{1 *}$, Sabina Spigner ${ }^{2}$, Vishal Swaminathan ${ }^{1}$, Seth Teplitsky ${ }^{1}$, Rosemary Frasso ${ }^{2}$

${ }^{1}$ Department of Urology, Sidney Kimmel Medical College, Thomas Jefferson University, Philadelphia, PA, ${ }^{2}$ College of Population Health, Thomas Jefferson University, Philadelphia, PA, USA

Paul.Chung@jefferson.edu, Sabina.Spigner@jefferson.edu,Vishal.Swaminathan@jefferson.edu, Seth.Teplitsky@jefferson.edu, Rosemary.Frasso@jefferson.edu

Short title: Transgender Non-Binary Urological Care Seeking

Keywords: Transgender, non-binary, urology, care, qualitative

Acknowledgements: The research was supported by the Jefferson Emerging Medical Research Support Junior Faculty Award from Thomas Jefferson University and a Pilot Research Project Program Grant from the World Professional Association for Transgender Health (PHC).

\author{
*Corresponding Author \\ Paul H. Chung, MD \\ Assistant Professor \\ Department of Urology \\ Sidney Kimmel Medical College \\ Thomas Jefferson University \\ 1025 Walnut St. Ste. 1100 \\ Philadelphia, PA 19107 \\ Tel: $215-955-6961$ \\ Fax: 215-923-1884 \\ Email: paul.chung@jefferson.edu
}


OBJECTIVE: To describe perspectives and experiences related to urology care-seeking of transgender and non-binary (TGNB) individuals assigned male at birth.

MATERIALS AND METHODS: This HIPAA-compliant study was IRB approved and followed Consolidated Criteria for Reporting Qualitative Studies (COREQ) guidelines. Through semistructured interviews, perspectives and experiences of individuals related to urology care-seeking were explored. Open-ended questions were designed to elicit a range of responses rather than quantifiable data. Thematic codes were developed and explicitly defined. Codes pertaining to patient experiences were assessed and described.

RESULTS: Twenty-five TGNB individuals assigned male at birth were interviewed. Participants reported an array of factors that informed and inhibited care-seeking, factors that framed individual urologic care experiences, and their overall impression of the healthcare system's ability to effectively and respectfully serve the TGNB population. Specifically, participants reported that prior negative healthcare experiences dissuaded them from seeking care such as feeling discriminated against and having a lack of trust in providers. Additionally, participants reported feeling a need and responsibility to "educate" providers on both their medical needs and psychosocial experiences. Participants were also unclear how best to identify "trans-friendly" urologists who are culturally competent and have appropriate medical knowledge.

CONCLUSIONS: 
Transgender and non-binary (TGNB) individuals face significant barriers to care for unique healthcare needs. TGNB participants described care avoidance and reported experiences of healthcare discrimination. These data highlight the importance for urologists to understand the perspectives and historical experiences of these individuals who may seek urological care. 


\section{INTRODUCTION}

There are approximately 1 million people in the United States, transgender and non-binary (TGNB), who have a gender identity which differs from the sex they were assigned at birth [1]. While transgender individuals may identify as masculine or feminine within the gender binary, non-binary individuals may identify as, but not limited to, having two or more genders, no gender, moving between genders (gender fluid), or in-between genders. It is well documented that the TGNB population faces significant barriers to care which further widens the already present health disparity gap between this population and their cis-gender counterparts (i.e. people who identify with a gender that corresponds to their birth sex) [2,3]. Patient-related barriers to care may include insurance coverage, ability to take time off from work, and access to transportation. Non-patient related barriers are equally influential and may include a lack of providers who are comfortable with interacting with TGNB patients or who are knowledgeable about providing care to this population [4-6].

As the number of TGNB individuals and use of gender affirming (confirming) surgery increases, it is important for urologists to have direct exposure to the perspectives and historical experiences of these individuals who may seek their care [7]. Although TGNB individuals have unique healthcare needs that may require specialized urology-related transition care, such as gender affirming bottom surgery (i.e. phalloplasty, vaginoplasty, metoidioplasty, or orchiectomy), many of these individuals, like their cis counterparts will need to see a general urologist for conditions like kidney stones, voiding dysfunction, sexual dysfunction, prostate cancer screening, and other urological cancer interventions. TGNB individuals may be reluctant to seek care for these issues due to fear of stigma, misgendering, and being treated disrespectfully. 
The aim of the study is to characterize the perspectives and experiences related to urology care-seeking of TGNB individuals assigned male at birth. This qualitative study was designed to utilize semi-structured interviews to gather an array of perspectives rather than collect empirical data. Therefore, the information from this study is transferrable and intended to shed light on the experiences of this demographic and open the door for quantifiable and generalizable studies which we are developing. We hope these studies will increase and improve care by increasing awareness and decreasing stigma in urological settings.

\section{MATERIALS AND METHODS}

\section{Study Overview}

This HIPAA-compliant study was approved by the IRB and followed COREQ (Consolidated Criteria for Reporting Qualitative Studies) guidelines [8]. Using an inductive qualitative approach, semi-structured interviews were performed to explore the perspectives and experiences of seeking and obtaining urological care. This was not a quantitative study and was not intended to weigh responses. The study was designed and conducted by a team consisting of an attending urologist (PHC), an MPH student (SS), medical students (VS, ST), and a qualitative research scientist with more than 15 years of experience $(\mathrm{RF})$, who trained the team and supervised data collection and analysis.

An interview guide (Appendix 1) of open-ended questions was designed to elicit a range of responses rather than quantifiable data. The guide included a series of questions asking participants to describe prior healthcare visits, urinary issues, and sexual issues. As common in this approach, the interview guide was piloted by the team, and minor modifications were made after conducting the first 10 interviews. 


\section{Data Collection}

Inclusion criteria included identifying as transgender or non-binary, assigned male at birth, and age 18 or older. Participants were recruited by convenience by posting flyers at local outpatient clinics and community centers known to be frequented by TGNB individuals and through a chain-referral sampling approach, often deployed when researching sensitive topics and hard to reach populations from May 2019 to July 2019 [9]. Phone interviews were conducted by SS and lasted approximately 45 to 60 minutes. Participants gave verbal consent and received a $\$ 40$ debit card to compensate for their time. Interviews were audio-recorded, transcribed verbatim, and de-identified by an independent third party. Transcripts were checked for accuracy by SS and VS.

\section{Data Analysis \& Coding}

A constant-comparison approach was used by evaluating completed interviews before later interviews were conducted. A sample size of 25 was considered sufficient to achieve saturation, meaning that later interviews did not generate new codes $[10,11]$. The study team developed a code book to guide the data analysis. Codes (Appendix 2) were developed in two ways: a priori (informed by a review of relevant literature and the interview guide) and through line-by-line reading of a subsample of interview transcripts. Each code was given an explicit definition to ensure coding accuracy and improve intercoder reliability [12]. Two members of the research team (SS, VS) independently coded all 25 transcripts. Five of the 25 transcripts were double-coded to ensure coding accuracy. Coding was facilitated by NVivo12 Software (QRS International, Doncaster, Australia). Throughout the coding process, coding discrepancies were identified through the software and resolved at team meetings. 
Intercoder reliability was calculated in NVivo12 by using the $\kappa$ coefficient. Complete agreement in coding correlates with a mean $\kappa$ value of 1 , near perfect agreement with a mean $\kappa$ value of 0.81 to 0.99 , substantial agreement with a mean $\kappa$ value of 0.61 to 0.80 , and moderate agreement with a mean $\kappa$ value of 0.41 to 0.60 [13]. After coding was complete, the team met, reviewed coding output, and organized the findings into thematic categories. Demographic data were also collected and summarized to describe the study population.

\section{RESULTS}

Twenty-five TGNB individuals assigned male at birth with an average age of 36 years (range 23-67) completed an interview. Twelve (60\%) individuals reported having undergone gender affirming bottom surgery and 13 (87\%) were on hormone replacement therapy (HRT). Analysis of intercoder reliability for this study revealed near perfect agreement (mean $\kappa=0.99$; range, 0.71-1.00). This result was supported by percentage of agreement analysis, which yielded a mean of 99.9\% (range, 95\%-100\%) agreement between coders for all codes. Interview responses were coded and organized into three thematic categories describe below and supported by participant quotations.

Patient Perspectives: Participants shared their perspectives on experiences that frame and influence their healthcare encounters (Table 1).

Trust in Providers - Participants reported trust in a healthcare provider (HCP) was dependent on the provider's ability to demonstrate relevant medical knowledge and was enhanced when a participant was made to feel they had autonomy over their bodies and they were trusted to know what was best for themselves. Participants felt more comfortable trusting their HCP in spaces that were dedicated to LGBTQ+ (lesbian, gay, bisexual, transgender, queer/questioning, with the 
plus sign signifying inclusion of other gender identities and orientations that are not specifically covered) health and known for being culturally competent. Another talked about purposefully seeking a safe space that feels inclusive and accepting and where providers have experience providing care to LGBTQ+ individuals.

Confidence in Providers - Respondents discussed feeling anxious when visiting new healthcare providers or clinics because they often did not know what level of knowledge and competency the clinicians and staff had in TGNB health. Interviewees also explained that they enter the healthcare space with a lack of confidence when HCPs express not knowing about specificities of care for TGNB individuals. For example, one participant was concerned when their doctor admitted that they did not know about the risks involved with gender affirming surgery and HRT.

Prior General Traumatic Experiences - Several participants mentioned experiencing traumatic events in the past outside of healthcare which contributed to their interactions with healthcare professionals. Two participants discussed having prior sexual trauma. Another respondent talked about being frustrated that the doctor did not understand the trauma that is often experienced in society by TGNB individuals.

Barriers - Many participants expressed that they avoid or have trouble seeking care due to financial and logistical barriers. Those with insurance commented on the difficulties of receiving coverage for certain procedures due to gender discrepancies between the chart and their legal paperwork. Those without insurance talked about the difficulties of finding a provider who would care for them and about not being able to access care as a result. People also reported difficulty acquiring approval for surgeries due to the required paperwork from mental health professionals and other providers. 
Healthcare Experiences: Participants described challenges they have faced in the healthcare space (Table 2).

Misgendering - Participants reported being frustrated with their providers for using incorrect pronouns and their "deadnames" (given name prior to transition) rather than their chosen names despite being corrected multiple times. They mentioned that staff would often not comply with their requests to have a note added to their files regarding their correct pronouns and name. Other healthcare experiences of being misgendered that were stated included being placed in the wrong gendered waiting room for surgery and having a mismatch between medical documentation and reported gender identity.

Educating Providers - Many interviewees talked about needing to educate the providers and staff in clinics about their identity to improve the care that they received. Some interviewees reported needing to explain nuances regarding TGNB health including details about surgeries and HRT. One participant stated that they feel obligated to educate their providers to protect the wellbeing of future patients.

Clinic Experiences - Some interviewees described engaging with staff as positive citing feeling comfortable when staff used affirming language and preferred pronouns and names. Other interviewees discussed noticing clinical staff looking confused when interacting with them. One participant recanted a time when they were asked invasive questions related to their gender identity when they were seeking care for a hand laceration. Another talked about having to complete intake forms with binary language that were not inclusive to TGNB patients.

Patient Needs: Participants described specific ways to improve their healthcare experiences (Table 3). 
Knowledgeable Providers - Interviewees talked about finding a provider with the knowledge and training to provide them with TGNB-specific health care. Some respondents described their providers' medical knowledge related to TGNB health as lacking and feeling as though their providers are oblivious about health care for the TGNB population. Some mentioned avoiding seeking care from doctors who did not have experience with patients who had undergone gendering affirming surgery. Others discussed feeling comfortable seeking care from providers who worked at an LGBTQ+ clinic or who had been recommended to them by friends and online reviews.

Culturally Competent Providers - Negative experiences relating to lack of provider cultural competency included interactions where providers did not understand the social challenges related to being TGNB in terms of safety and being out in public. Interviewees with providers who they deemed culturally competent mentioned that they felt professionally supported by the provider because they would use affirming language and ask about gender identity, name, and pronouns. One participant described cultural competency in TGNB health as being able to treat the patient "as a person and not just as a problem to solve."

Compassionate Providers - Some participants described their providers as compassionate, supportive, respectful, and affirming. Others mentioned that their providers were interested in discussing their goals as patients and open to discussing all options for their care. However, other participants described providers' uncompassionate attitudes as threatening, standoffish, and uncomfortable. They mentioned being mistreated and threatened by their HCPs and not feeling supported.

Balancing Treatments and Priorities - Some participants mentioned being frustrated that they could not access the benefits of HRT without encountering side effects, such as diminished 
libido and an impacted ability to elicit and maintain erections when applicable. Participants also cited surgical recovery time as a source of conflict between desire for a vaginoplasty and prioritizing quality of life. Further, some discussed moments when there was a mismatch between what their health priorities were versus what their HCPs wanted for them. One participant talked about feeling pressured to consider sperm preservation before starting HRT despite previously stating that they were not interested.

\section{DISCUSSION}

As the number of TGNB individuals and use of gender affirming surgery increases, it important for urologists to appreciate the perspectives and historical experiences of these individuals who may seek urological care [7]. In this study, TGNB individuals shared important insights into the urological care experience which serve as an important opportunity to improve services and to inform training for urologists and staff. Although several individuals reported feeling more comfortable in an LGBTQ+ specific clinic, it is unreasonable for these specialized clinics be the only place to seek competent care [14].

Provider knowledge was a dominant theme reported by the participants and prior studies $[3,4]$. Respondents reported decreased confidence and negative experiences with providers with limited understanding of TGNB health. Not all providers understand that identifying as TGNB falls on a spectrum. TGNB individuals desire different gender characteristics and may not desire the same hormonal, surgical, or therapeutic transitions. For example, erectile function may be important to some TGNB, who may consequently seek treatment for erectile dysfunction, but not all. Participants were fatigued from being their own advocate and needing to educate physicians about their desired procedures. While some patients may be amenable to serving as a source of 
education, providers must recognize that not all patients feel this way nor should the onus fall on the patient to educate them. Unfortunately, these accounts regarding need for improved provider competency are common. In 2015, the United States Transgender Survey (USTS) was completed by 27,715 respondents and is the largest report of TGNB experiences [15]. When respondents to the USTS were able to see a provider, $33 \%$ reported at least one negative experience related to being TGNB including having to teach the provider about TGNB people.

Provider cultural competence, or lack thereof, was an important issue identified by participants in our study and in prior studies [16]. Participants reported that providers need to be conscious that their TGNB identity may not always be relevant to the chief complaint and that it is inappropriate to ask questions regarding gender identity not related to the care requested. Fifteen percent of respondents from the USTS reported being asked invasive or unnecessary questions about being TGNB unrelated to their health visit [15]. Providers need to be cognizant of misgendering which occurs when a person is referred to using a word, such as a pronoun, that does not correctly reflect the gender with which the person identifies.

Issues with cultural competency are not limited to providers and staff alone but also refer to the lack of inclusive environments and safe spaces at clinics and hospitals $[4,14]$. Twentythree percent of respondents from the USTS reported not seeking care at all when needed due to fear of being mistreated in the clinic [15]. Many opportunities exist for clinics and hospitals to provide "safe space" environments for all patients. Such opportunities include personnel training in gender-identity competency, providing gender neutral bathrooms, forms not limited to binary pronouns, and minimizing incorrect name or gender on template notes that may be perpetuated in the medical record. Medical documentation needs to account for non-binary genders, and the medical community needs to recognize that changing a name and gender can lead to logistical, 
administrative, and legal challenges. Only $11 \%$ of participants from the USTS had their desired name and gender on all of their identifications and this present study also reflected this reality [15].

Clinical exposure to TGNB health and education are lacking [6,17]. A substantial portion of urology residency programs provide no education on this topic and the ones that do have largely variable content [18]. Experience with TGNB patients is regionally variable, with the highest rates of experience in the Western and North Central portions of the United States [19]. One study evaluated the knowledge of all members of a urology clinic and identified that the majority of respondents had received some form of training, but only $11.5 \%$ considered it satisfactory [20]. Multiple efforts to educate medical providers have been initiated by the American Urological Association (AUA) and associated societies. These include a workgroup, a telemedicine webcast for medical students, an instructional course at the AUA annual meeting, and contributions to the AUA Update Series and Core Curriculum.

Respondents were frustrated by providers who did not understand the emotional and physical journey that they may have experienced and desired providers who are empathetic, supportive, respectful, and affirming. TGNB individuals are at a high risk for attempting suicide in their lifetime (40\% of respondents from the USTS) and face high rates of unemployment [15]. Nearly $30 \%$ of respondents from the USTS experienced homelessness at some point in their lives and nearly half $(47 \%)$ of respondents had been sexually assaulted. Medical visits and seemingly routine physical exams may be intimidating and remind individuals of prior abuse, potentially leading to avoidance of care. It is important for clinicians to recognize the prevalence of trauma in this demographic and be able to deliver trauma-informed care by promoting a culture of safety, empowerment, and healing [21]. Informed providers will be able to recognize the effects 
of trauma and resist re-traumatizing patients. Specific examples may include reducing power dynamics, use of gender inclusive diagrams, using less gendered terms, and limiting the number of providers a patient has to see [22].

Several limitations exist with this study which explored a range of care seeking experiences and barriers to the care of TGNB individuals using a systematic qualitative approach. Open-ended questions were designed to elicit a range of responses and gather an array of perspectives through conversation rather than quantifiable data. Participants were recruited by convenience through flyers and a chain-referral sampling approach with participants largely from a single metropolitan area and was limited to individuals assigned male at birth, therefore, the findings may not be transferable to all TGNB people. Although some responses were regarding non-urological care, an understanding of prior experiences will help to prepare urologists to provide an improved experience to individuals seeking urological care. The intent of the study is to shed light on the experiences of this demographic and open the door for quantifiable and generalizable studies in the future.

\section{CONCLUSIONS}

TGNB care has gained increased attention by urological providers. Shedding light on the care seeking experience and barriers to care for this community may foster awareness among providers, influence educational initiatives, inform practice level interventions to create more welcoming and inclusive spaces, and ultimately increase access to, and improve care for the population. 


\section{REFERENCES}

1. Meerwijk EL and Sevelius JM: Transgender Population Size in the United States: a Meta-Regression of Population-Based Probability Samples. Am J Public Health 2017; 107: e1-e8.

2. Christian R, Mellies AA, Bui AG, et al.: Measuring the Health of an Invisible Population: Lessons from the Colorado Transgender Health Survey. J Gen Intern Med 2018; 33 : 1654-60.

3. Puckett JA, Cleary P, Rossman K, et al.: Barriers to Gender-Affirming Care for Transgender and Gender Nonconforming Individuals. Sex Res Social Policy 2018; 15: 48-59.

4. Safer JD, Coleman E, Feldman J, et al.: Barriers to healthcare for transgender individuals. Curr Opin Endocrinol Diabetes Obes 2016; 23: 168-71.

5. Socias ME, Marshall BD, Aristegui I, et al.: Factors associated with healthcare avoidance among transgender women in Argentina. Int J Equity Health 2014; $13: 81$.

6. Fraser L and Knudson G: Education Needs of Providers of Transgender Population. Endocrinol Metab Clin North Am 2019; 48: 465-77.

7. Nolan IT, Kuhner CJ and Dy GW: Demographic and temporal trends in transgender identities and gender confirming surgery. Transl Androl Urol 2019; 8: 184-90.

8. Tong A, Sainsbury P and Craig J: Consolidated criteria for reporting qualitative research (COREQ): a 32-item checklist for interviews and focus groups. Int J Qual Health Care 2007; 19: 349-57.

9. Penrod J, Preston DB, Cain RE, et al.: A discussion of chain referral as a method of sampling hard-to-reach populations. J Transcult Nurs 2003; 14: 100-7. 
10. BF C and WL M: Doing qualitative research: research methods for primary care. Newbury Park, California, Sage, 1992.

11. Saunders B, Sim J, Kingstone T, et al.: Saturation in qualitative research: exploring its conceptualization and operationalization. Qual Quant 2018; 52: 1893-907.

12. BG G and AL S: The discovery of grounded theory: strategies for qualitative research. Chicago, Illinois, Aldine, 1967.

13. Viera AJ and Garrett JM: Understanding interobserver agreement: the kappa statistic. Fam Med 2005; 37: 360-3.

14. Whitlock BL, Duda ES, Elson MJ, et al.: Primary Care in Transgender Persons. Endocrinol Metab Clin North Am 2019; 48: 377-90.

15. James SE, Herman JL, Rankin S, et al.: The Report of the 2015 U.S. Transgender Survey. Washington, DC, National Center for Transgender Equality, 2016.

16. Eisenberg ME, McMorris BJ, Rider GN, et al.: "It's kind of hard to go to the doctor's office if you're hated there." A call for gender-affirming care from transgender and gender diverse adolescents in the United States. Health Soc Care Community 2020.

17. Coutin A, Wright S, Li C, et al.: Missed opportunities: are residents prepared to care for transgender patients? A study of family medicine, psychiatry, endocrinology, and urology residents. Can Med Educ J 2018; 9: e41-e55.

18. Morrison SD, Dy GW, Chong HJ, et al.: Transgender-Related Education in Plastic Surgery and Urology Residency Programs. J Grad Med Educ 2017; 9: 178-83.

19. Dy GW, Osbun NC, Morrison SD, et al.: Exposure to and Attitudes Regarding Transgender Education Among Urology Residents. J Sex Med 2016; 13: 1466-72. 
20. Teplitsky S, Counsilman M and Chung PH: An Assessment of an Outpatient Urology Clinic's Compassion, Comfort and Knowledge of Transgender Care. Urol Prac 2020; 7 : 61-67.

21. Brezing C, Ferrara M and Freudenreich O: The syndemic illness of HIV and trauma: implications for a trauma-informed model of care. Psychosomatics 2015; 56: 107-18.

22. Hall SF and DeLaney MJ: A Trauma-Informed Exploration of the Mental Health and Community Support Experiences of Transgender and Gender-Expansive Adults. J

Homosex 2019: 1-20. 